\title{
Anticancer Effect and Mechanism of Hydroxygenkwanin in Oral Squamous Cell Carcinoma
}

\author{
Yi-Chao Huang ${ }^{1,2}$, Po-Chuan Lee ${ }^{1}$, Jane Jen Wang ${ }^{3}$ and Yi-Chiung Hsu ${ }^{1 *}$ \\ ${ }^{1}$ Department of Biomedical Sciences and Engineering, National Central University, Taoyuan, Taiwan, ${ }^{2}$ Department of Medical \\ Laboratory, Taoyuan Armed Forces General Hospital, Taoyuan, Taiwan, ${ }^{3}$ The Department of Nursing, School of Nursing, \\ National Taipei University of Nursing and Health Sciences, Taipei, Taiwan
}

OPEN ACCESS

Edited by:

Cheng-Chia Yu,

Chung Shan Medical

University, Taiwan

Reviewed by:

Shih-Yin Chen,

China Medical University, Taiwan

Chia-Hung Yen,

Kaohsiung Medical University, Taiwan

*Correspondence:

Yi-Chiung Hsu

syicncu@g.ncu.edu.tw

Specialty section:

This article was submitted to Head and Neck Cancer, a section of the journal

Frontiers in Oncology

Received: 05 August 2019 Accepted: 02 September 2019 Published: 18 September 2019

Citation:

Huang $Y-C$, Lee $P$-C, Wang JJ and Hsu Y-C (2019) Anticancer Effect and Mechanism of Hydroxygenkwanin in

Oral Squamous Cell Carcinoma.

Front. Oncol. 9:911.

doi: 10.3389/fonc.2019.00911
The incidence and mortality of oral squamous cell carcinoma (OSCC) are high, and the number of oral cancers had risen in the world. However, chemotherapy drugs have numerous side effects. There is an urgent requirement to develop a novel drug that can be used to treat oral cancer. Hydroxygenkwanin (HGK) is a nature flavonoid extracted from Daphne genkwa Sieb. et Zucc. (Thymelaeaceae). Previous studies had demonstrated that HGK exhibits anticancer effect, but the effect is still unclear in oral cancer. HGK inhibited cell growth dose-dependently in SAS and OCEM1 cells. The functional enrichment analysis showed the significant pathway in cellular movement, cell cycle and cellular growth and proliferation. We further demonstrated the HGK induced the cell cycle arrest by flow cytometry and inhibited colony formation ability and cell movement. The western blot showed that HGK induced cell cycle arrest through p21 activation and caused intrinsic cell apoptosis pathway. HGK inhibited the cell invasion and migration through down-regulation vimentin. HGK might be an effective natural product for oral cancer therapy.

Keywords: oral cancer, RNA sequencing, apoptosis, cell cycle, migration, invasion

\section{INTRODUCTION}

Oral cancer is a lethal disease worldwide with a 5-year survival rate of around $50 \%$ and oral squamous cell carcinoma (OSCC) accounts for $90 \%$ of all oral cancer types found in the mouth, tongue, and lips (1). The oral cancer is highly related to the exposure of risk factors such as betel nut chewing, smoking, alcohol drinking, and virus infection (2). From 2004 to 2015, the oral cancer incidence keeps increasing in Taiwan. Surgery, radiotherapy, and chemotherapy are the three main treatments intended to stop or eliminate the spread of oral cancer (3). However, chemotherapy compounds would cause normal cells damage. In order to reduce the side effect of treatment in oral cancer, identification of the new potentially anticancer compounds is necessary. Hydroxygenkwanin (HGK) is purification from Daphne genkwa plant. Daphne genkwa (yuanhua in Chinese), is one kind of Daphne plants (Thymelaeaceae) distributed in Europe, Taiwan and China. Daphne genkwa is a commonly used traditional Chinese medicine (TCM) known to have purgative and diuretic effects (4). The daphnane diterpenoid from the flowers of Daphne genkwa, has been demonstrated a potential anti-cancer effect in human non-small cell lung cancer (NSCLC) cells and anti-gioma $(5,6)$. The Genkwa flos flavonoids significantly decreased arthritis through antioxidant and hemorheological modulatory mechanisms in animal study (7). However, the systematic studies of HGK, the compound from Daphnis Genkwa Flo, in oral cancer is limited. We systematically study the gene expression profile in OECM1 and SAS cells treated with HGK. Furthermore, we investigate the anti-cancer mechanism of HGK in oral cancer cells. 


\section{MATERIALS AND METHODS}

\section{Cell Culture}

HGK was purchased from Shanghai BS Bio-Tech Co., Ltd (Shanghai, China) and was dissolved in dimethyl sulfoxide (DMSO) (Merck, Darmstadt, Germany) as a stock solution of $100 \mathrm{mM}$ and stored at $-20^{\circ} \mathrm{C}$ before use. SAS is a human tongue squamous cell carcinoma from the Japanese Collection of Research Bioresources (Tokyo, Japan) (2). OECM1, a human gingival squamous carcinoma cells, derived according to previous study (2). Two cell lines were authenticated by short tandem repeat analysis and cultured in Dulbecco's modified Eagle's medium (DMEM) supplemented with $10 \%$ fetal bovine serum (FBS), $1.2 \mathrm{~g} / \mathrm{L}$ sodium bicarbonate, $0.5 \mathrm{mM}$ sodium pyruvate, and $2.5 \mathrm{mM}$ L-glutamine. Chemical compounds, culture medium and FBS were purchased from Life Technologies (Grand Island, NY, USA).

\section{Phenotypic Examination}

Phenotypes including cell proliferation (MTT assay), clonogenic ability, migration, and invasion assay were done as described previously $(2,8)$.

\section{RNA Sequencing}

The total RNA samples are first treated with DNase I to degrade any possible DNA contamination. Then the mRNA is enriched by using the oligo (dT) magnetic beads. Mixed with the fragmentation buffer, the mRNA is fragmented into short fragments. Then the first strand of cDNA is synthesized by using random hexamer-primer. Buffer, dNTPs, RNase $\mathrm{H}$ and DNA polymerase I are added to synthesize the second strand. The double strand cDNA is purified with magnetic beads. End reparation and $3^{\prime}$-end single nucleotide $\mathrm{A}$ (adenine) addition is then performed. Finally, sequencing adaptors are ligated to the fragments. The fragments are enriched by PCR amplification. During the QC step, Agilent 2100 Bioanaylzer and ABI Step One Plus Real-Time PCR System are used to qualify and quantify of the sample library. The library products are ready for sequencing via Illumina HiSeq 4000 or other sequencer when necessary.

\section{Differential Expression Analysis}

Primary sequencing data that produced by Illumina Hiseq 4000 (San Diego, CA), called as raw reads, is subjected to quality control (QC) to determine if a resequencing step is needed (Using Trimmomativ v0.33). After QC, raw reads are filtered into clean reads which will be aligned to the reference sequences (mm10 for mouse) by Bowtie2 v2.2.6. QC of alignment
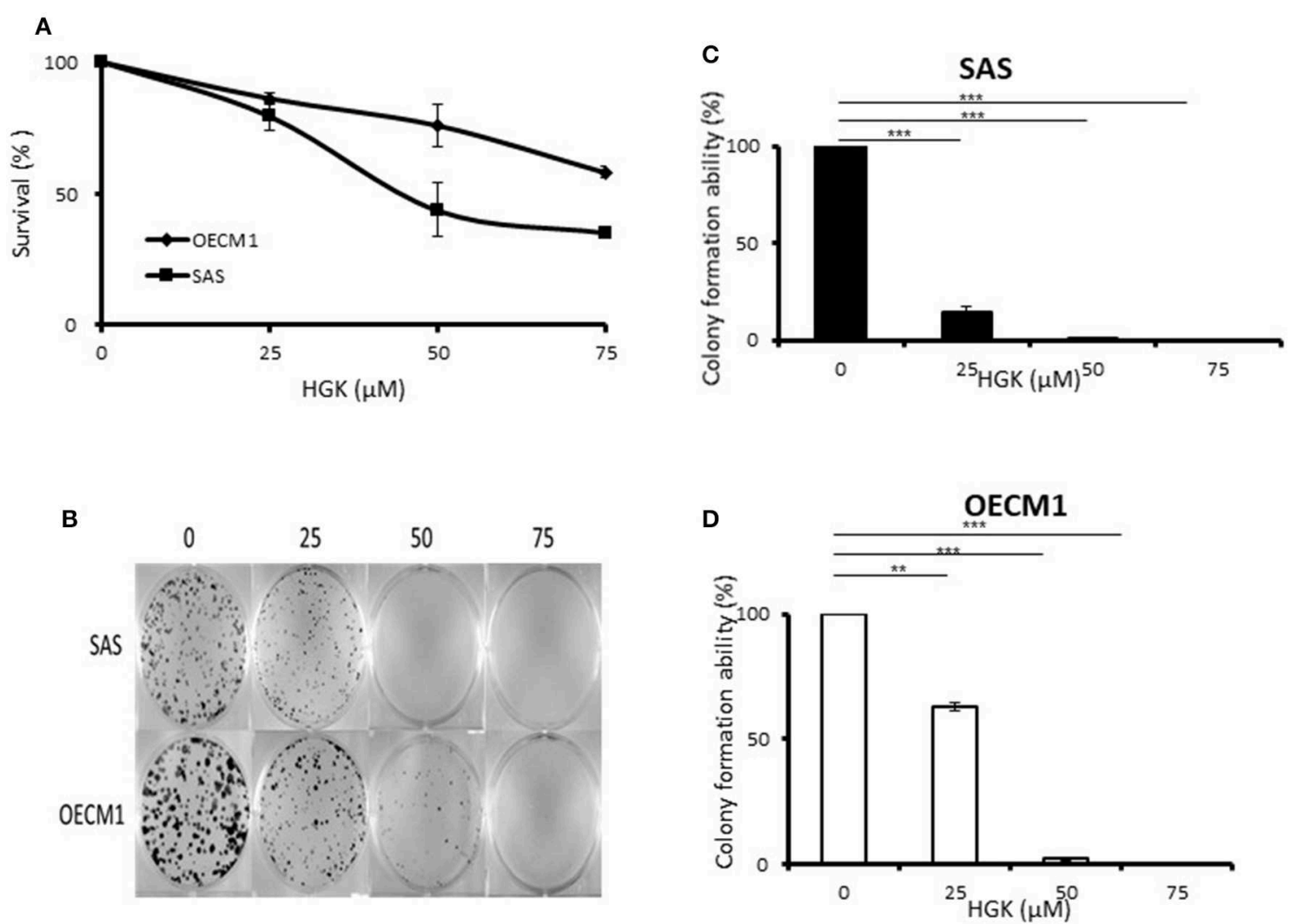

FIGURE 1 | Effects of HGK on the cell survival assay and colony formation of SAS and OECM1 cells. SAS and OECM1 cells were treated with HGK for $24 \mathrm{~h}$ in different concentrations (0, 25, 50, $75 \mu \mathrm{M})$. (A) MTT assay (B) The images showing colony formation assay in two cancer cells treated with HGK. (C) The quantitative analysis of colony numbers in SAS cells (D) in OECM1 cells. ${ }^{* *} P<0.01$ and ${ }^{* * *} P<0.001$. 


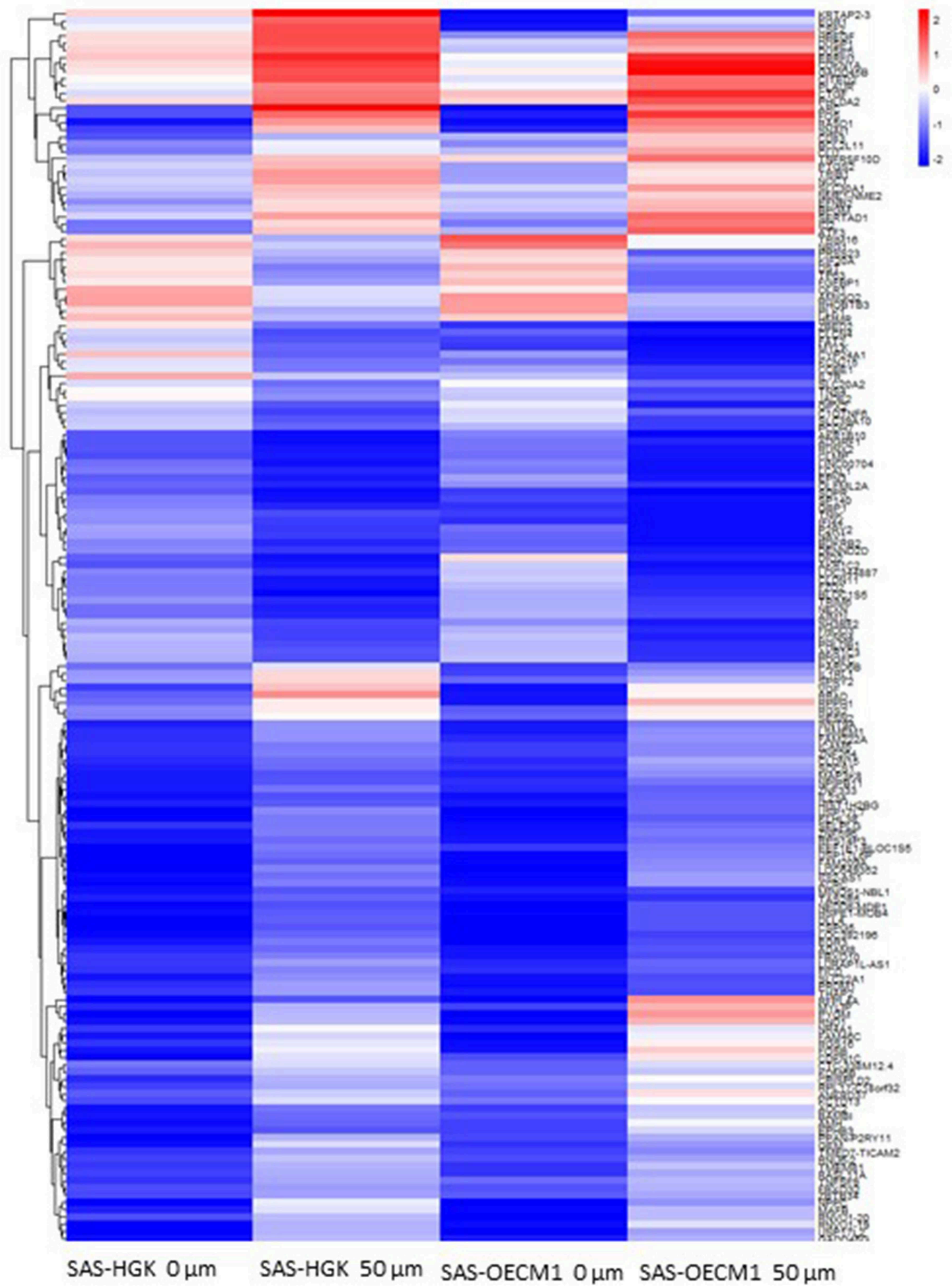

FIGURE 2 | Heatmap showing differential gene expression level with $50 \mu \mathrm{M}$ HGK treatment for $24 \mathrm{~h}$. Red indicating upregulation, and blue representing downregulation. 
is performed to determine if resequencing is needed. The alignment data is utilized to calculate distribution of reads on reference genes and mapping ratio. Once all of the clean reads mapped to reference sequences, we're using "RSEM (RNA-seq by Expectation Maximization)" for calculating read raw count and normalized quantification from each sample. As we got the read quantification data, we could continue various different comparisons. The statistical tool we selected is "EBSeq v1.16.0" which may be used to identify DEGs (differential expressed genes). The RNAseq data were publicly available on NCBI's Sequence Read Archive (SRA) database (Bioproject: PRJNA559691).

\section{Functional Enrichment}

The pathway enrichment were analyzed by the Ingenuity Pathway Analysis (IPA) (QIAGEN company, Redwood City, CA, USA), and Gene set enrichment analysis (GSEA) (9).

TABLE 1 | Molecular and cellular functions analysis of differential gene expression in HGK-treated oral cancer cells.

\begin{tabular}{llc}
\hline Name & p-value range & \#Molecules \\
\hline Cellular movement & $2.32 \mathrm{E}-04-2.39 \mathrm{E}-15$ & 68 \\
Cell death and survival & $2.34 \mathrm{E}-04-7.60 \mathrm{E}-12$ & 74 \\
Cell cycle & $2.35 \mathrm{E}-04-1.09 \mathrm{E}-11$ & 87 \\
Cellular growth and proliferation & $2.35 \mathrm{E}-04-1.09 \mathrm{E}-11$ & 78 \\
DNA replication & $2.80 \mathrm{E}-11-2.80 \mathrm{E}-11$ & 22
\end{tabular}

\section{Cell Cycle Analysis}

Cell cycle analysis was performed as previously described (10). Briefly, cells were fixed in $-20^{\circ} \mathrm{C}$ absolute ethanol for $4 \mathrm{~h}$ and resuspended in PBS having $20 \mu \mathrm{g} / \mathrm{ml}$ ribonuclease A. After incubating at $37^{\circ} \mathrm{C}$ for $30 \mathrm{~min}$, propidium iodide $100 \mu \mathrm{g} / \mathrm{ml}$ was added to samples. Cell cycle was examined by flow cytometry (BD FACSCalibur TM system, Becton-Dickinson).

\section{Western Blotting}

Western blotting was performed as described previously (2). Antibodies against poly (ADP-ribose) polymerase (PARP) (Asp214), caspase 9, phosphor-H2A.X (ser139), E-cadherin, and Vimentin were purchased from Cell Signaling (Temecula, CA, USA). Anti-p21 and $\beta$-actin were purchased from Santa Cruz Biotechnology (Santa Cruz, CA, USA).

\section{Statistics}

Student's $t$-test was completed by the Statistical Package for the Social Sciences version 12.0 (SPSS, Inc). Differences between the variables were considered significant for $p$-values $<0.05$.

\section{RESULTS}

\section{Effects of HGK on the Cell Survival Assay and Colony Formation in SAS and OECM1 Cells}

To determine the ability of HGK to inhibit cell growth and colony formation in two cell lines, cells were incubated in the absence or presence of increasing concentrations of HGK for $24 \mathrm{~h}$. The growth and colony formation of SAS and OECM1 cells were reduced after exposure to $25-75 \mu \mathrm{M}$ HGK for $24 \mathrm{~h}$ (Figure 1).
A

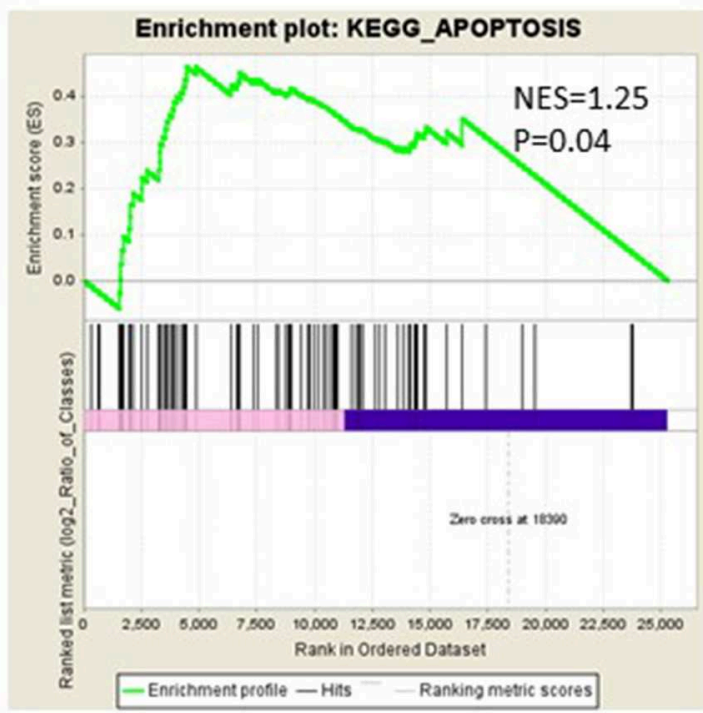

B

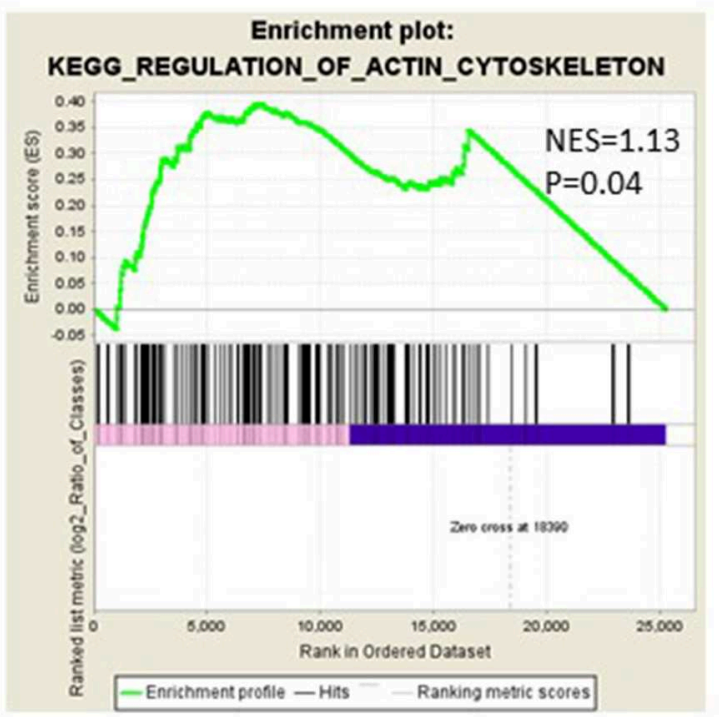

FIGURE 3 | Gene Set Enrichment Analysis (GSEA) enrichment plot involved in (A) apoptosis (B) in regulation of actin cytoskeleton. 

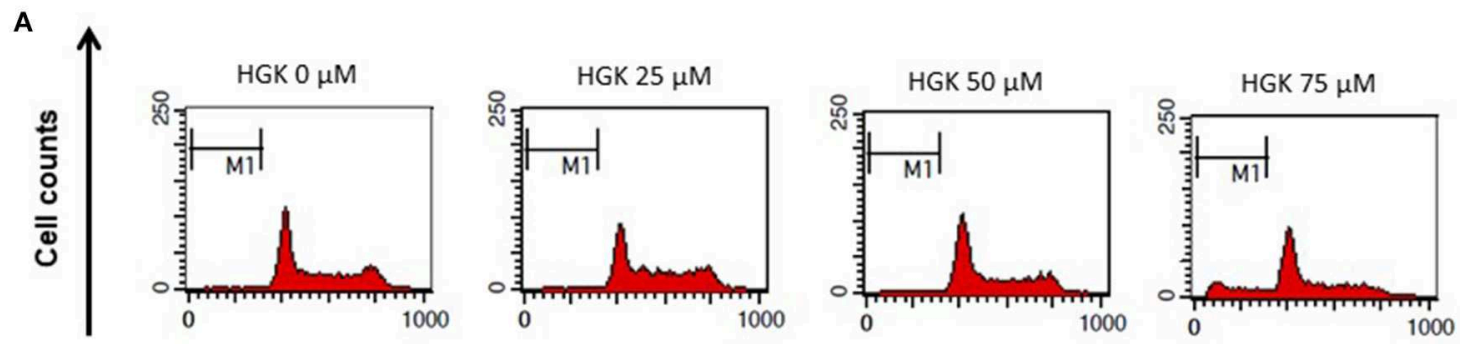

Propidium iodide (PI)

SAS

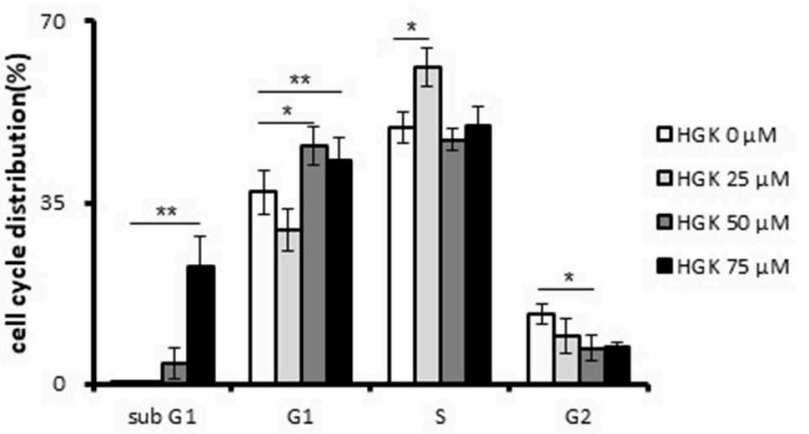

B
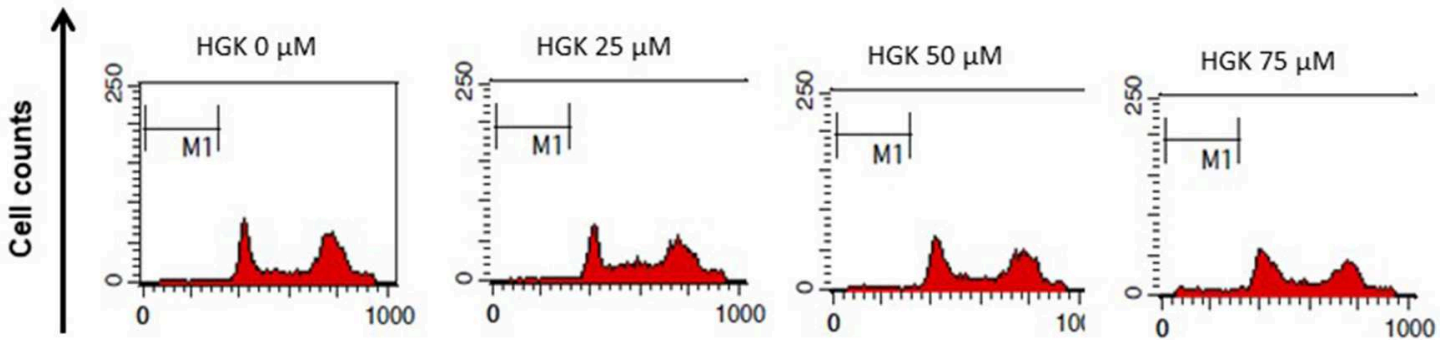

Propidium iodide (PI)

\section{OECM1}

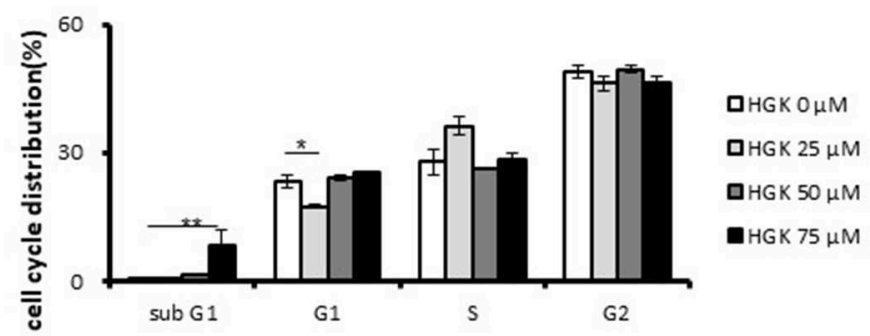

FIGURE 4 | Effects of HGK on the cell cycle progression in SAS and OECM1 cells. SAS cells (A) and OECM1 cell (B) were treated with $0,25,50,75 \mu M$ for $24 \mathrm{~h}$. The results are shown as the mean \pm S.E. of three independent experiments. ${ }^{\star} P<0.05$ and ${ }^{\star *} P<0.01$. 


\section{A}
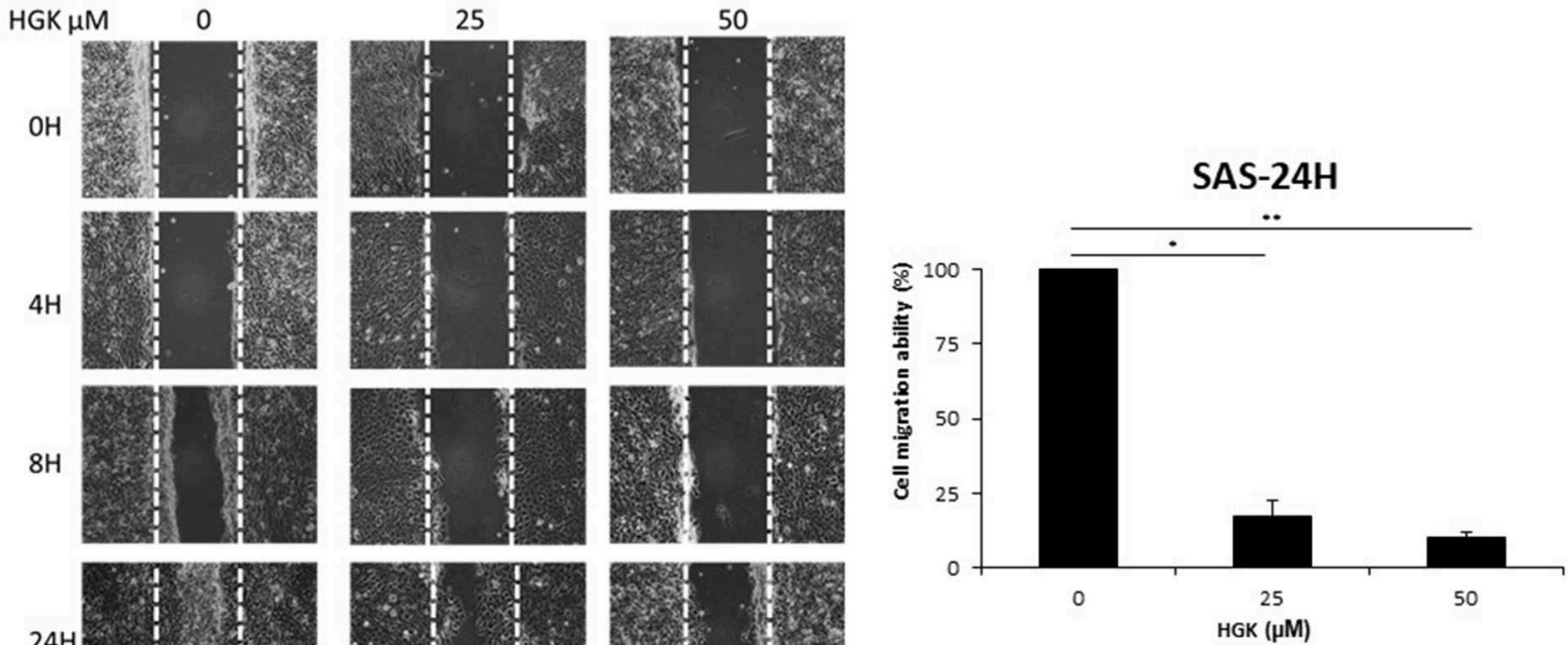

B
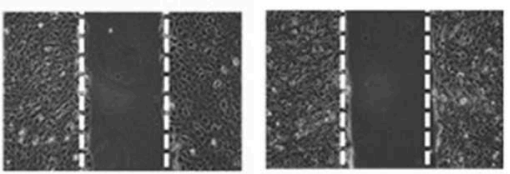

$8 \mathrm{H}$
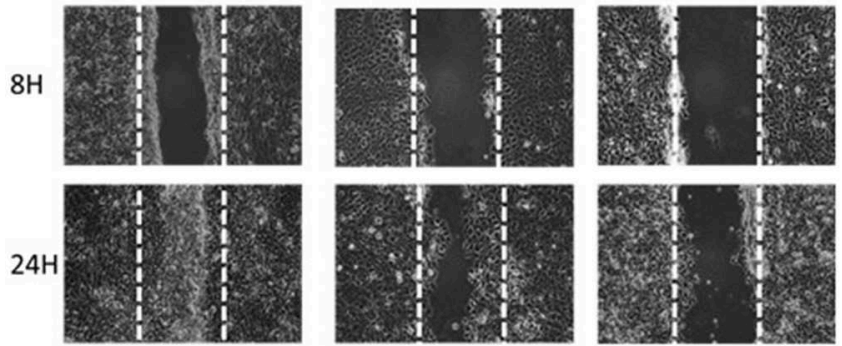

HGK $(\mu \mathrm{M})$
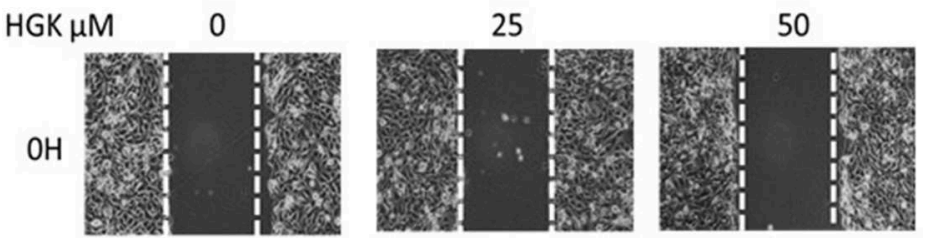

OECM1-24H
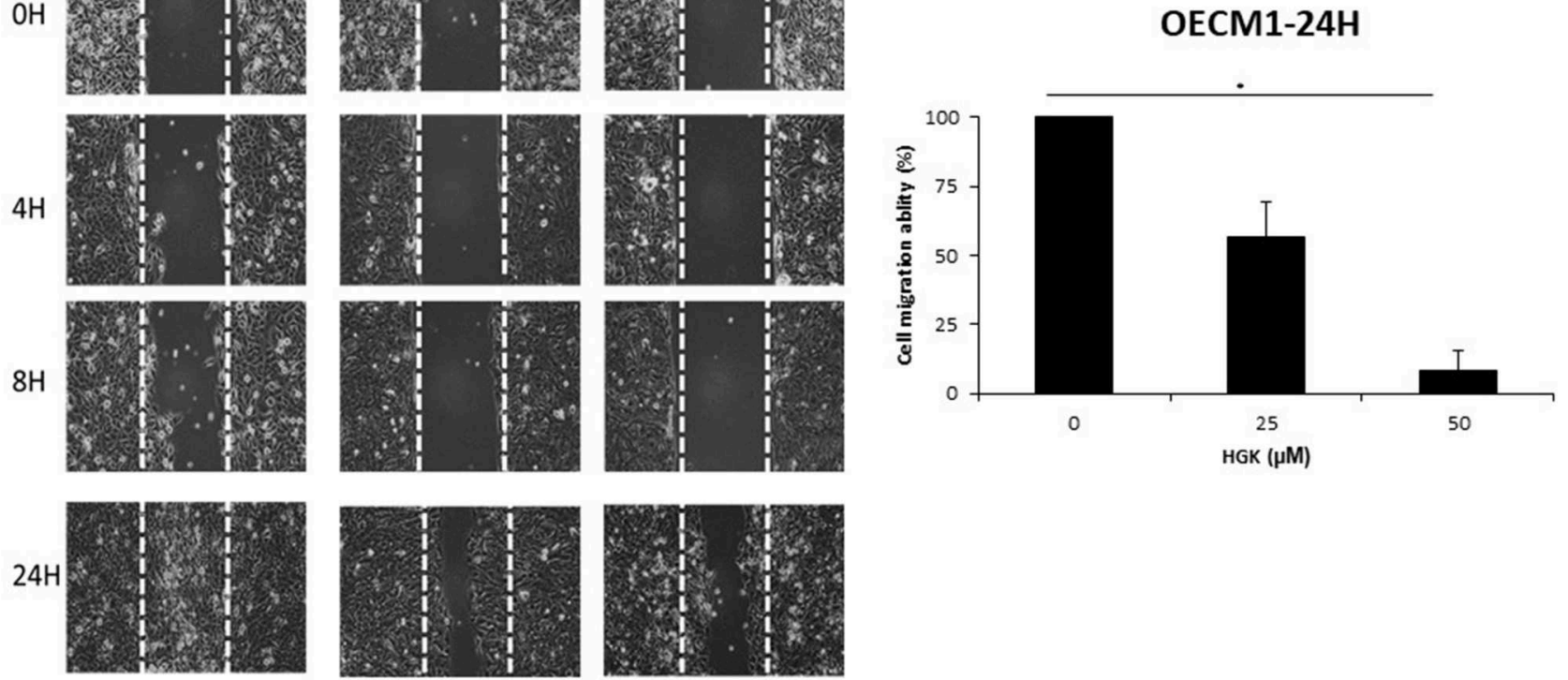

FIGURE 5 | Effects of HGK on cell migration in SAS (A) and OECM1 (B) cells. SAS and OECM1 cells were treated with 0, 25, 50, $75 \mu \mathrm{M} \mathrm{HGK} \mathrm{for} 24 \mathrm{~h}$. The results are shown as the mean \pm S.E. of three independent experiments. ${ }^{*} P<0.05$ and ${ }^{\star *} P<0.01$. 
The inhibition activity of HGK on cell survival assay and colony formation of SAS cells was more potent than on OECM1 cells.

\section{Functional Enrichment Analysis of Differential Gene Expression Genes After HGK Treatment}

We identified the differential gene expression genes from RNAseq data. A filtering criterion of FPKM value is more than 1 (11), and $\log 2$ fold change was greater than two in both SAS and OECM1 datasets. The sign of change must be consistent in both cell lines. A total of 274 genes were significant in the SAS dataset and 969 genes in the OECM1 dataset (Table S1). 170 genes were consistent change in both cell lines (Figure 2). Table 1 showed the most significant molecular and cellular function of differential expression genes after HGK treatment in SAS and OECM1 cells. The final consistency 170 genes were listed in the Table S2. The cellular functions affected by HGK were involved in the cellular movement, cell cycle and cell proliferation (Table 1). GSEA shows a statistically significant difference between biological samples association with apoptosis and regulation of actin cytoskeleton functions

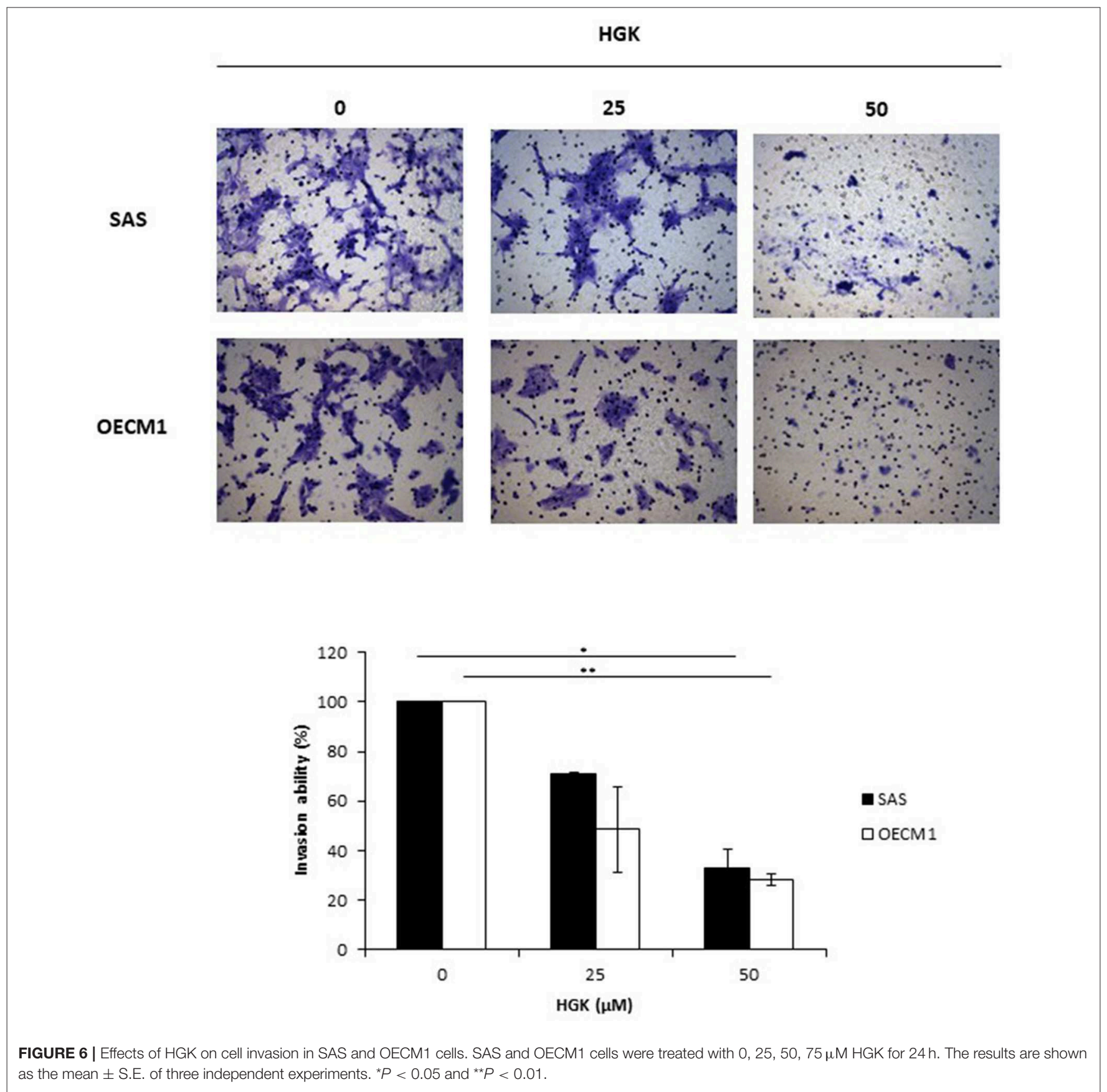


(Figure 3). Differential expression and pathway analysis in HGK treatment oral cancer cells indicated the cell cycle, cell survival and cell movement pathways were potential mechanisms.

\section{Effects of HGK on the Cell Cycle Progression in SAS and OECM1 Cells}

We further examine the HGK biological function according to the results of functional enrichment. For investigation of the effect of HGK on cell cycle progression, cells were stained by propidium iodide (PI) and measured by flow cytometry at $24 \mathrm{~h}$ following 25-75 $\mu \mathrm{M}$ HGK drug treatments. In Figure 4, as compare to control $(0 \mu \mathrm{M})$, HGK dose dependently increased the percentage of sub $G_{0} / G_{1}$ phase SAS and OECM1 cells, and reduced the cells in $\mathrm{G} 2 / \mathrm{M}$ phases of SAS cells but not OECM1 cells. 50 and $75 \mu \mathrm{M}$ HGK increased the percentage of $\mathrm{G}_{0} / \mathrm{G}_{1}$ phase SAS cells, but $25 \mu \mathrm{M}$ HGK reduced both of the cells in $\mathrm{G}_{0} / \mathrm{G}_{1}$ phases and concomitantly increased the percentage of $S$ phase cells (Figure 4).

\section{Effects of HGK on Cell Motion in SAS and OECM1 Cells}

To investigate the HGK effect on SAS and OECM1 cell migration, we used wound healing assay. The cell migration was obviously reduced in SAS and OECM1 cells at 8 and $24 \mathrm{~h}$ after 25 and $50 \mu \mathrm{M}$ HGK treatment (Figure 5). To investigate the HGK effect on SAS and OECM1 cell invasion, we used transwell invasion assay. As shown in Figure 6, the invasion was significantly reduced in 25 and $50 \mu \mathrm{M}$ HGK treated- SAS and OECM1 cells.

\section{Effects of HGK on Cell Cycle, Apoptosis, and EMT Regulatory Proteins in SAS and OECM1 Cells}

In a previous result, we confirmed that HGK increased the percentage of SAS cells but not OECM1 cells in G0/G1 phase. In order to identify the molecular involved in the HGK effect of the G0/G1 phase, SAS and OECM1 cells were treated with 50 and $75 \mu \mathrm{M}$ HGK for $24 \mathrm{~h}$, and the cells were harvested for Western blotting. We observed the up-regulation of p21 in SAS cells after HGK treatment, but downregulation of p21 in OECM1 cells (Figure 7). p21 acts at the checkpoint in the cell cycle at the G1 phase. Western blot indicated that cell cycle regulated p21 might play an important role in in HGK-induced cell cycle arrest. HGK dose dependently increased the percentage of sub $\mathrm{G}_{0} / \mathrm{G}_{1}$ phase SAS and OECM1 cells (Figure 4). We further examined the apoptosis effect by using of H2AX Phosphorylation (12) and cleaved PARP cleaved caspase 9 (13). Western blot analyses of pH2AX, cl-PARP and cleaved caspase 9 in both HGK treated-SAS and OECM1 cells. The epithelial-mesenchymal transition (EMT) provides cells with migration and invasion abilities (14). We measured the e-cadherin (epithelial marker), and vimentin (mesenchymal markers) in HGK-treated cells. Vimentin showed significantly decreased between the HGKtreated and control groups, but not significant up-regulation of E-cadherin (Figure 7).

\section{DISCUSSION}

Natural compound and their related moieties played an important role in drug discovery and development (15). HGK

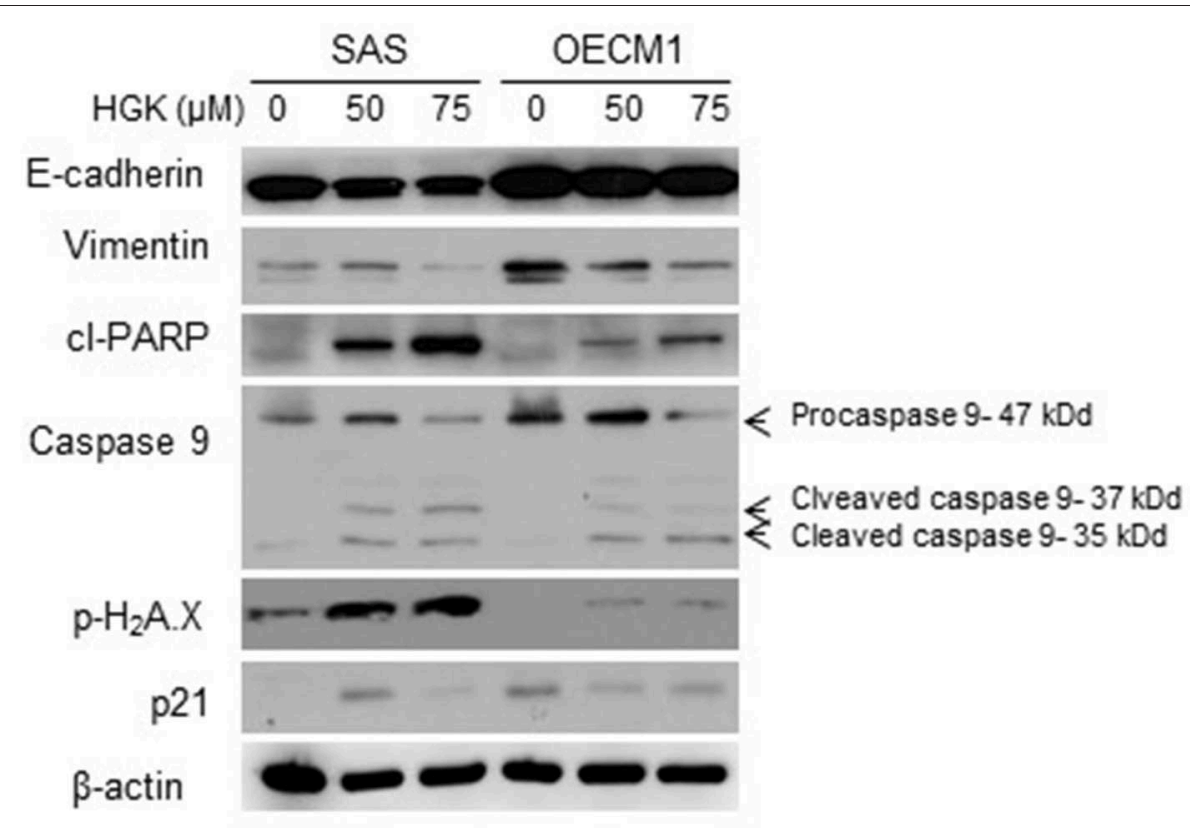

FIGURE 7 | Western blot analysis of cell cycle, apoptosis, and EMT regulatory proteins after treatment with HGK in SAS and OECM1 cells. $\beta$-actin was used as the internal marker. 
was obtained from novel flavonoid extracted from Daphne genkwa and induced cancer cell toxicity, cell cycle arrest, apoptosis and EMT signaling pathways in different types of OSCC cell lines (OECM1 and SAS). We demonstrated that HGK significantly dose-dependently inhibited the rate of cell growth in OECM1 and SAS cells. SAS cells were higher sensitivity to HGK than OECM1 cells (Figure 1). The SAS cell line was high malignancy and metastasis derived from a poorly differentiated squamous cell carcinoma. The OECM-1 cell line was less malignancy established from a Taiwanese patient (16). Nextgeneration sequencing technologies are being widely applied in biomedical research (17). Systems for transcriptome profiling provided the insight into the specific biological pathways. Increases in G0/G1 phase cell cycle arrest with increasing doses of HGK treatments in SAS cells, but not OECM1 (Figure 4). Increase of the sub-G1 phase with various doses of HGK in both SAS and OECM1 cells. Western blot results showed that HGK activated the protein levels of p21 in SAS cells, but decrease in OECM1 cells (Figure 7). The results were consistent with cell cycle analysis. The G1-S transition in cell cycle is driven mainly by cyclin-dependent kinase (CDK) 2 that is controlled by abundance of CDK inhibitors: p21 (18). p21 was a key cell cycle regulator that arrests cells in the G1 and G2 phases. Therefore, p21 proteins were involved in HGK-induced the cell cycle arrest in SAS cells. HGK induce the cell death of OSCC cells by apoptosis. The mechanisms of apoptosis are involving two main apoptotic pathways: the extrinsic and the intrinsic pathway (19). HGK induced cell apoptosis through the intrinsic pathway by activation of cleave Caspase 9, and cleave PARP (Figure 7). Histone H2AX phosphorylation was sensitive marker for DNA double-strand breaks (20). We demonstrated that HGK induce DNA damage in both two cell lines. Moreover, H2AX phosphorylation also an essential role in cell cycle arrest regulated by p21 and cell growth (21). HGK induced cell cycle arrest not only through p21 activation but also H2AX phosphorylation. Decrease of E-cadherin and gain of vimentin promoted tumor migration, leading to higher metastatic risk of head and neck squamous cell carcinoma patients (22). E-cadherin was an important therapeutic target for designing anti-tumor drugs (6). Figure 7 showed that SAS1 and OECM1 cells did not loss the Ecadherin expression. Therefore, HGK inhibited the cell invasion and migration through down-regulation vimentin, not increase in E-cadherin.

In this study, we provided the bioinformatics analysis and biological functions experiments of HGK-treated oral cancer cells. Our results showed HGK had anti-cancer activities through multiple mechanisms including inhibition of cellular movement,

\section{REFERENCES}

1. Markopoulos AK. Current aspects on oral squamous cell carcinoma. Open Dent J. (2012) 6:126-30. doi: 10.2174/1874210601206 010126

2. Chen CY, Chiou SH, Huang CY, Jan CI, Lin SC, Hu WY, et al. Tid1 functions as a tumour suppressor in head and neck squamous cell carcinoma. J Pathol. (2009) 219:347-55. doi: 10.1002/path.2604 cell cycle arrest and blocking cell proliferation in oral cancer cells. In conclusion, this study provided evidence that HGK was a potential natural anti-tumor compound for squamous cell carcinoma. However, further pharmacological and investigations in vivo are required.

\section{DATA AVAILABILITY}

The datasets generated in the study can be found in the SRA database (https://www.ncbi.nlm.nih.gov/sra) using the following accession numbers: STUDY: PRJNA559691, SAMPLE: OECM_HGK (SAMN12551609), EXPERIMENT: 7 (SRX6701569), RUN: OECM1-4_HHFT2DSXX_L4_R1.fastq.gz (SRR9953208), SAMPLE: OCEM_control (SAMN12551608), EXPERIMENT: 5 (SRX6701570), RUN: OECM13_HHFT2DSXX_L4_R1.fastq.gz (SRR9953207), SAMPLE: SAS_HGK (SAMN12551607), EXPERIMENT: 3 (SRX6701571), RUN: SAS-2_HHFT2DSXX_L4_R1.fastq.gZ (SRR9953206), SAMPLE: SAS_control (SAMN12551606), EXPERIMENT: 1 (SRX6701572), RUN: SAS-1_HHFT2DSXX_L4_R1. fastq.gz (SRR9953205).

\section{AUTHOR CONTRIBUTIONS}

$\mathrm{Y}-\mathrm{CHu}$ and P-CL performed the experiments and analyzed the data. Y-CHu, Y-CHs, and JW drafted the manuscript. Y-CHs contributed to conception and design of the study. All authors have read and approved the final manuscript.

\section{FUNDING}

This study was supported by grants from Ministry of Science and Technology, Taiwan (MOST-107-2314-B-008-002) and Taoyuan Armed Forces General Hospital, Taiwan (AFTYGH-10858). We thank Dr. Chi-Yuan Chen (Chang Gung University of Science and Technology) for providing technical support and intellectual discussions in this study.

\section{SUPPLEMENTARY MATERIAL}

The Supplementary Material for this article can be found online at: https://www.frontiersin.org/articles/10.3389/fonc. 2019.00911/full\#supplementary-material

Table S1 | The gene expression profiles in SAS and OECM1 treated with HGK

Table S2 | The consistency expression genes. 
5. Kang JI, Hong JY, Lee HJ, Bae SY, Jung C, Park HJ, et al. Anti-tumor activity of yuanhuacine by regulating AMPK/mTOR signaling pathway and actin cytoskeleton organization in non-small cell lung cancer cells. PLOS ONE. (2015) 10:e0144368. doi: 10.1371/journal.pone.0144368

6. Song Y, Ye M, Zhou J, Wang ZW, Zhu X. Restoring E-cadherin expression by natural compounds for anticancer therapies in genital and urinary cancers. Mol Ther Oncolytics. (2019) 14:130-8. doi: 10.1016/j.omto.2019.04.005

7. Zhang CF, Zhang SL, He X, Yang XL, Wu HT, Lin BQ, et al. Antioxidant effects of Genkwa flos flavonoids on Freunds adjuvant-induced rheumatoid arthritis in rats. J Ethnopharmacol. (2014) 153:793-800. doi: 10.1016/j.jep.2014.03.046

8. Chen CY, Yang SC, Lee KH, Yang X, Wei LY, Chow LP, et al. The antitumor agent PBT-1 directly targets HSP90 and hnRNP A2/B1 and inhibits lung adenocarcinoma growth and metastasis. J Med Chem. (2014) 57:677-85. doi: $10.1021 / j m 401686$ b

9. Subramanian A, Tamayo P, Mootha VK, Mukherjee S, Ebert BL, Gillette MA, et al. Gene set enrichment analysis: a knowledge-based approach for interpreting genome-wide expression profiles. Proc Natl Acad Sci USA. (2005) 102:15545-50. doi: 10.1073/pnas.0506580102

10. Wang TH, Chan CW, Fang JY, Shih YM, Liu YW, Wang TV, et al. 2-O-Methylmagnolol upregulates the long non-coding RNA, GAS5, and enhances apoptosis in skin cancer cells. Cell Death Dis. (2017) 8:e2638. doi: $10.1038 /$ cddis.2017.66

11. Mortazavi A, Williams BA, McCue K, Schaeffer L, Wold B. Mapping and quantifying mammalian transcriptomes by RNA-Seq. Nat Methods. (2008) 5:621-8. doi: 10.1038/nmeth.1226

12. Plesca D, Mazumder S, Almasan A. DNA damage response and apoptosis. Methods Enzymol. (2008) 446:107-22. doi: 10.1016/S0076-6879(08)01606-6

13. Kitazumi I, Tsukahara M. Regulation of DNA fragmentation: the role of caspases and phosphorylation. FEBS J. (2011) 278:427-41. doi: 10.1111/j.1742-4658.2010.07975.x

14. Lu W, Kang Y. Epithelial-mesenchymal plasticity in cancer progression and metastasis. Dev Cell. (2019) 49:361-74. doi: 10.1016/j.devcel.2019.04.010

15. Butler MS. The role of natural product chemistry in drug discovery. J Nat Prod. (2004) 67:2141-53. doi: 10.1021/np040106y
16. Chuang CY, Chang CP, Lee YJ, Lin WL, Chang WW, Wu JS, et al. PRMT1 expression is elevated in head and neck cancer and inhibition of protein arginine methylation by adenosine dialdehyde or PRMT1 knockdown downregulates proliferation and migration of oral cancer cells. Oncol Rep. (2017) 38:1115-23. doi: 10.3892/or.2017.5737

17. Han Y, Gao S, Muegge K, Zhang W, Zhou B. Advanced applications of RNA sequencing and challenges. Bioinform Biol Insights. (2015) 9(Suppl. 1):29-46. doi: 10.4137/BBI.S28991

18. Sherr CJ, Roberts JM. CDK inhibitors: positive and negative regulators of G1phase progression. Genes Dev. (1999) 13:1501-12. doi: 10.1101/gad.13.12.1501

19. Elmore S. Apoptosis: a review of programmed cell death. Toxicol Pathol. (2007) 35:495-516. doi: 10.1080/01926230701320337

20. Sharma A, Singh K, Almasan A. Histone H2AX phosphorylation: a marker for DNA damage. Methods Mol Biol. (2012) 920:613-26. doi: 10.1007/978-1-61779-998-3_40

21. Fragkos M, Jurvansuu J, Beard P. H2AX is required for cell cycle arrest via the p53/p21 pathway. Mol Cell Biol. (2009) 29:2828-40. doi: $10.1128 / \mathrm{MCB} .01830-08$

22. Nijkamp MM, Span PN, Hoogsteen IJ, van der Kogel AJ, Kaanders JH, Bussink J. Expression of E-cadherin and vimentin correlates with metastasis formation in head and neck squamous cell carcinoma patients. Radiother Oncol. (2011) 99:344-8. doi: 10.1016/j.radonc.2011.05.066

Conflict of Interest Statement: The authors declare that the research was conducted in the absence of any commercial or financial relationships that could be construed as a potential conflict of interest.

Copyright (c) 2019 Huang, Lee, Wang and Hsu. This is an open-access article distributed under the terms of the Creative Commons Attribution License (CC BY).

The use, distribution or reproduction in other forums is permitted, provided the original author(s) and the copyright owner(s) are credited and that the original publication in this journal is cited, in accordance with accepted academic practice. No use, distribution or reproduction is permitted which does not comply with these terms. 